\title{
Percepção de fisioterapeutas sobre aspectos bioéticos em cuidados paliativos
}

Fabíola Alves Alcântara

Universidade Federal do Rio de Janeiro, Rio de Janeiro/RJ, Brasil.

\begin{abstract}
Resumo
O objetivo do estudo é investigar a percepção de fisioterapeutas sobre aspectos bioéticos que envolvem o atendimento a pacientes sem possibilidades terapêuticas de cura. Trata-se de estudo transversal, descritivo, com profissionais do Conselho Regional de Fisioterapia e Terapia Ocupacional da Primeira Região. Foi aplicado questionário tipo survey, baseado nos princípios de autonomia, beneficência, não maleficência e justiça. Os resultados mostraram que a maioria dos profissionais atua com pacientes sem possibilidade terapêutica de cura, considera extremamente relevante o processo de tomada de decisão durante o atendimento, respeita a vontade do paciente, busca defender o princípio da beneficência e reconhece a importância da bioética e das discussões éticas na formação acadêmica. Conclui-se que os fisioterapeutas buscam tomar decisões de modo ético, mas há conflito de valores no que se refere a princípios como autonomia e beneficência.
\end{abstract}

Palavras-chave: Bioética. Ética. Cuidados paliativos. Pessoal de saúde. Fisioterapia.

\section{Resumen}

Percepción de fisioterapeutas sobre los aspectos bioéticos que involucran los cuidados paliativos El objetivo del estudio es investigar la percepción de fisioterapeutas sobre los aspectos bioéticos que involucran el cuidado de pacientes sin posibilidades terapéuticas de curación. Se realizó un estudio descriptivo transversal con profesionales del Consejo Regional de Fisioterapia y Terapia Ocupacional de la Primera Región. Se aplicó un cuestionario de encuesta basado en los principios de autonomía, beneficencia, no maleficencia y justicia. Los resultados mostraron que la mayoría de los profesionales trabajan con pacientes sin posibilidad terapéutica de cura, consideran de extrema relevancia el proceso de toma de decisiones durante el cuidado, respetan la voluntad del paciente, buscan defender el principio de beneficencia y reconocen la importancia de la bioética y de las discusiones éticas en la educación académica. Se concluye que los fisioterapeutas buscan tomar decisiones de forma ética, pero existe un conflicto de valores en torno a principios como la autonomía y la beneficencia.

Palabras clave: Bioética. Ética. Cuidados paliativos. Personal de salud. Fisioterapia.

\section{Abstract}

\section{Perception of physiotherapists on bioethical aspects in palliative care}

The study investigates physiotherapists' perception on bioethical aspects involving care to patients without therapeutic possibilities of cure. This is a cross-sectional, descriptive study with professionals from the Regional Council of Physical Therapy and Occupational Therapy of the First Region. A survey questionnaire was applied based on the principles of autonomy, beneficence, non-maleficence, and justice. Results showed that most professionals work with patients with no therapeutic possibility of cure, consider the decision-making process during care to be extremely relevant, respect the patient's will, seek to defend the principle of beneficence, and recognize the importance of bioethics and ethical discussions in academic education. In conclusion, physiotherapists seek to make ethical decisions, but there is a conflict of values regarding principles such as autonomy and beneficence.

Keywords: Bioethics. Ethics. Palliative care. Health personnel. Physical therapy specialty. 
Com o aumento do número de casos de doenças crônico-degenerativas, cânceres, demências e outras patologias, os cuidados paliativos aparecem como exemplo de assistência integral ao indivíduo, atendendo a seus desejos e necessidades. $\mathrm{Na}$ rotina clínica, é comum que fisioterapeutas enfrentem questões éticas, o que se intensifica no tratamento de pacientes sem possibilidades terapêuticas de cura, que envolve ainda outros tipos de questionamento.

Compreender as incertezas envolvidas nesse contexto e entender como os profissionais se posicionam diante do conflito é fundamental para o embasamento ético da assistência. Segundo Gracia, o estudo de tais dilemas não tem por objetivo resolvê-los em definitivo, mas, antes de tudo, compreendê-los de forma adequada, entendendo os argumentos das distintas posturas, seu peso e suas limitações, de modo tal que possamos ter elementos suficientes para a realização de juízos ponderados e prudentes ${ }^{1}$.

Considerando que a tomada de decisão clínica do fisioterapeuta é importante para o cuidado do paciente sem possibilidades terapêuticas de cura e que estudos brasileiros sobre a temática ainda são escassos, aponta-se a relevância deste estudo. Seu propósito é investigar aspectos bioéticos que envolvem o atendimento fisioterapêutico desses pacientes, na busca por entender os critérios éticos em que os profissionais se baseiam.

Vários questionamentos permeiam a prática clínica, sobretudo no que se refere à terminalidade da vida. Para o fisioterapeuta, questões como limitação terapêutica e falta de recursos são vividas como dilemas éticos. Mas o que esse profissional considera quando precisa tomar decisões envolvendo pacientes sem possibilidade terapêutica de cura? Este estudo visa colaborar para a compreensão desse processo.

\section{Materiais e método}

Trata-se de estudo transversal descritivo do tipo survey, realizado com profissionais cadastrados no Conselho Regional de Fisioterapia e Terapia Ocupacional da Primeira Região (Crefito 1) ${ }^{2}$. Os dados foram coletados pela internet mediante questionário semiestruturado nos estados de Alagoas, Paraíba, Pernambuco e Rio Grande do
Norte, a partir de parceria estabelecida com o Crefito 1 entre dezembro de 2017 e janeiro de 2018.

O Crefito 1 é autarquia federal de última instância recursal estabelecida pela Lei $6.316 / 1975^{3}$, que criou o Conselho Federal (Coffito) e os conselhos regionais de fisioterapia e terapia ocupacional. Essas entidades foram formadas para supervisionar o exercício dos profissionais da área ${ }^{2}$. Dentre suas funções está uniformizar quesitos éticos, científicos e sociais e fiscalizar empresas que prestam assistência fisioterapêutica ${ }^{3}$. $O$ Crefito 1 abrange quatro estados do Nordeste: Alagoas, Paraíba, Pernambuco e Rio Grande do Norte ${ }^{2}$.

Segundo o artigo $3^{\circ}$ da Resolução Coffito $424 / 2013^{4}$, a profissão de fisioterapeuta só pode ser exercida por aqueles que estiverem inscritos no Conselho Regional de seu local de atuação. Foram incluídos na amostra todos os profissionais registrados no Crefito 1 que aceitaram participar da pesquisa. Aqueles que não tinham correio eletrônico cadastrado no Conselho foram excluídos.

Os dados foram coletados a partir de questionário sobre aspectos bioéticos do atendimento fisioterapêutico a pacientes sem possibilidades terapêuticas de cura. $O$ instrumento foi construído com base no Código de Ética e Deontologia da Fisioterapia ${ }^{4}$, que converge em suas disposições com a abordagem principialista da bioética, enfatizando autonomia e beneficência. Também foram utilizados como referência os trabalhos de Barnitt ${ }^{5}$ e Renner, Goldim e Prati ${ }^{6}$.

As respostas foram apresentadas em escala Likert de cinco pontos (indo de 1, não relevante, até 5 , extremamente relevante), disponibilizando-se ainda espaço para sugestões e comentários. O questionário foi aplicado por meio do programa LimeSurvey, que permite reduzir possíveis vieses causados pela presença de entrevistadores, além de apresentar vantagens como baixo custo, fácil acesso e possibilidade de aplicação a distância. Os dados foram organizados em planilha do Microsoft Office Excel e analisados de acordo com os achados.

O instrumento foi encaminhado pelo Crefito 1 simultaneamente para todos os profissionais cadastrados, junto com carta-convite para participação na pesquisa, incluindo orientações quanto ao preenchimento do questionário e sua devolução. $O$ convite foi acompanhado de link protegido por senha que levava a uma página inicial com o termo de 
consentimento livre e esclarecido (TCLE). Tal documento precisava ser lido e aceito pelos participantes para que conseguissem acessar o questionário.

Ao terminar de preencher o instrumento, todos receberam uma cópia do TCLE via correio eletrônico. A pesquisa obedeceu aos critérios éticos estabelecidos na Resolução 466/2012 do Conselho Nacional de Saúde ${ }^{7}$. Os profissionais convidados foram devidamente informados sobre os objetivos e métodos do estudo, e os que aceitaram participar manifestaram seu consentimento.

\section{Resultados e discussão}

Trinta dias após o envio dos convites, foram respondidos apenas $50(0,3 \%)$ dos 17 mil questionários enviados. Do total de respondentes, 32 (64\%) eram do sexo feminino, 16 (32\%) do masculino e 2 (4\%) não declararam sexo. Tendência semelhante de maior participação de mulheres foi encontrada nos estudos de Pereira ${ }^{8}$ e Renner, Goldim e Prati ${ }^{6}$. De acordo com o Instituto Nacional de Estudos e Pesquisas Educacionais Anísio Teixeira ${ }^{9}$, em 2015 o número de concluintes do sexo feminino (548.682) em cursos de graduação no Brasil foi consideravelmente maior que do sexo masculino (367.681). Talvez a diferença na quantidade de retornos ao convite se deva a essa maior presença de mulheres no cenário acadêmico-profissional.

A média de idade dos participantes foi de 27,51 anos (24-46 anos), e os respondentes tinham se formado, em média, há cinco anos. Esse perfil contrasta com o da pesquisa de Guardia Mancilla e colaboradores ${ }^{10}$, em que a média de idade dos participantes ficou em 44,58 anos, com tempo de formação médio de 20 anos. Há semelhança, porém, com o estudo de Pereira ${ }^{8}$, em que $42 \%$ da amostra tinha se formado há até 5 anos.

Pernambuco foi o estado em que mais fisioterapeutas atuavam com pacientes sem possibilidades terapêuticas de cura. Dentre as unidades federativas consideradas na pesquisa, esta é a que apresenta maior número de instituições de ensino superior e maior população ${ }^{11}$.

A maioria dos fisioterapeutas era apenas graduada (44\%), seguida daqueles com pós-graduação lato sensu (32\%) e stricto sensu (24\%). Quanto às especialidades mais representativas, 32\% dos participantes disseram atuar na área traumato-ortopédica, e $16 \%$ em gerontologia. Os outros $52 \%$ estavam dispersos em outras especialidades. Em Pereira ${ }^{8}$, a área de atuação mais prevalente foi a ortopedia, enquanto em Renner, Goldim e Prati ${ }^{6}$ houve grande variação. Ambos os estudos, portanto, convergem com a presente pesquisa.

Quando perguntados se trabalhavam com pacientes sem possibilidades terapêuticas de cura, $72 \%$ responderam que sim, $22 \%$ que não e $6 \%$ não responderam a essa pergunta. A maioria dos respondentes, portanto, atua com cuidados paliativos.

Na segunda parte do questionário foi solicitado aos participantes que classificassem uma série de afirmativas em uma escala de relevância de 1 (não relevante) a 5 (extremamente relevante). Quanto à tomada de decisão durante $o$ atendimento fisioterapêutico, 54\% responderam que esta é extremamente relevante. Machado, Pessini e Hossne ${ }^{12}$ ratificam essa consideração quando destacam que o dia a dia de profissionais que atuam com pacientes em fim de vida é permeado por dilemas que requerem preparo para tomar decisões.

A maioria da amostra (68\%) também julgou extremamente relevante a discussão de conflitos éticos em equipe. Tratando do tema, Machado, Pessini e Hossne ${ }^{12}$ destacam que os cuidados paliativos exigem reflexões bioéticas para que as decisões da equipe sejam pautadas no respeito à autonomia e à dignidade do enfermo.

Sobre a vontade do paciente, $46 \%$ responderam que é extremamente relevante respeitá-la, ratificando mais uma vez o que propõem Machado, Pessini e Hossne ${ }^{12}$. Essa consideração da autonomia como importante para a prática profissional está de acordo com o Código de Ética e Deontologia da Fisioterapia ${ }^{4}$.

Além da autonomia, constatou-se o respeito ao princípio da beneficência, uma vez que $56 \%$ dos participantes o consideraram extremamente relevante, em postura que se pode considerar principialista ${ }^{13,14}$. Cabe apontar, entretanto, que o princípio da beneficência é aquele que mais costuma entrar em conflito com a autonomia. De um lado, o doente precisa ter suas decisões respeitadas e, de outro, a prática médica muitas vezes se contrapõe à vontade do paciente em nome da beneficência, recaindo em atitudes paternalistas ${ }^{15}$.

A inclinação principialista da amostra é confirmada pelo fato de que $42 \%$ dos participantes 
consideram extremamente relevante tomar decisões com base na ideia de justiça. Beauchamp e Childress salientam que nenhum princípio moral isoladamente tem capacidade de dar conta de todos os problemas de justiça ${ }^{16}$. Assim, entende-se que "decidir com justiça" é considerar todos os direitos do paciente, enquanto decidir com injustiça é privá-lo de seus direitos, falhando na distribuição dos benefícios.

No Brasil, o modelo bioético mais utilizado é o principialismo, fundamentado na autonomia, na beneficência, na não maleficência e na justiça ${ }^{15}$. Em revisão de literatura sobre artigos brasileiros que relacionam fisioterapia e bioética, Lorenzo e Bueno ${ }^{17}$ apontaram que todos os estudos analisados faziam referência à corrente principialista. Segundo Beauchamp e Childress, fundadores dessa corrente, os princípios são diretrizes gerais que deixam espaço para julgamento específico em casos especificos e que ajudam explicitamente no desenvolvimento de regras e linhas de ação mais detalhadas ${ }^{16}$. Tais princípios, portanto, não são absolutos, e não há hierarquia entre eles.

Pessini e Bertachini ${ }^{18}$ demonstraram a tendência principialista nos cuidados paliativos quando ressaltaram os preceitos estabelecidos pela Organização Mundial da Saúde para esse tipo de assistência. No questionário elaborado para a presente pesquisa foram contempladas perguntas relacionadas à teoria principialista sem que os conceitos da corrente fossem apresentados. No estudo de Renner, Goldim e Prati ${ }^{6}$, em que definições foram apresentadas, os profissionais não conseguiram correlacionar os princípios com a prática.

Quanto à falta de recursos financeiros disponíveis aos profissionais para conduzir a terapia do paciente, observaram-se resultados mais divididos: $25 \%$ dos respondentes consideraram esse ponto relevante, mas não houve consenso. Ressalta-se, no entanto, com base em pesquisas como a de Silva, Lima e Seidl ${ }^{19}$, que problemas financeiros podem impactar a atuação do profissional de saúde que lida com cuidados paliativos, interferindo em sua visão de justiça.

Em relação aos recursos financeiros dos pacientes, verifica-se resultado semelhante ao da questão anterior: $26 \%$ dos respondentes consideraram essa questão conflito ético importante, mas muitos a classificaram como pouco $(14 \%)$ ou não relevante (8\%). O resultado demonstra falta de reflexão sobre aspectos da bioética em parte significativa dos participantes, visto que a questão é, sim, relevante. Esse tipo de desconhecimento aponta para a desumanização do atendimento, com profissionais tendendo a valorizar o manejo de pacientes críticos e discutir a decisão clínica sem adentrar o universo subjetivo ${ }^{19}$.

Quando perguntados se a falta de recursos financeiros do hospital, clínica ou unidade de saúde seria um conflito ético, $42 \%$ responderam que esse ponto é extremamente relevante. Isso indica que os participantes da pesquisa percebem a responsabilidade financeira da instituição como mais importante em comparação com os recursos do profissional e do paciente.

No que diz respeito à comunicação com o paciente e ao respeito aos princípios bioéticos, foi perguntado aos participantes o quão relevante é dizer a verdade ao enfermo. A maior parte dos fisioterapeutas considerou essa questão um conflito ético extremamente relevante (46\%). Sobre o tema, Kovács ${ }^{20}$ afirma que não mais se admite discutir o dever de informar, que é parte essencial do processo de morrer com dignidade.

Outro conflito ético que pode surgir no dia a dia dos fisioterapeutas é a necessidade de limitar a eficiência de uma terapia em prol do aprendizado, questão considerada extremamente relevante por $44 \%$ dos participantes. Tal conflito também faz parte do processo de comunicação, sendo necessário que o profissional considere os princípios bioéticos na tomada de decisão, respeitando sempre os direitos do paciente, principalmente seu direito a morrer com dignidade ${ }^{19-21}$.

Em relação à prática fisioterapêutica com pacientes sem possibilidades terapêuticas de cura em ambiente hospitalar, 54\% consideraram esse conflito extremamente relevante. Nesse sentido, ratifica-se a importância do fisioterapeuta nos cuidados paliativos, contribuindo com a qualidade de vida de pacientes terminais e aumentando as possibilidades de uma morte digna.

Como apontam Silva, Lima e Seidl, a atuação desse profissional é fundamental em todo o processo saúde-doença, pois contribui para a promoção da saúde, tratamento, reabilitação e prevenção de agravos, bem como em cuidados paliativos, com ênfase na qualidade de vida, preceito importante incorporado ao novo Código de Ética e Deontologia 
da Fisioterapia (...). O fisioterapeuta que trabalha com cuidados paliativos usa ainda recursos para aliviar a dor. Para esse tipo de trabalho terá à disposição alguns procedimentos terapêuticos que poderão minorar a dor e o sofrimento do paciente e auxiliar no seu manejo ${ }^{22}$.

Oliveira, Bombarda e Moriguchi ${ }^{23}$ estudaram a fisioterapia em cuidados paliativos na atenção primária e perceberam que os profissionais ainda estão mais voltados à reabilitação. Entretanto, a inserção na atenção primária poderia colaborar de forma significativa para o controle de sintomas e melhora da qualidade de vida dos pacientes.

Ainda sobre a importância do fisioterapeuta nos cuidados paliativos, mas mais especificamente sobre $o$ atendimento domiciliar, $52 \%$ dos respondentes consideraram essa questão extremamente relevante. Silva, Lima e Seidl ${ }^{19}$ ratificam a importância do fisioterapeuta no cuidado domiciliar a pacientes sem possibilidades terapêuticas de cura, salientando, contudo, a importância de preparar melhor esses profissionais com base na bioética, tendo em vista que o ambiente familiar também traz conflitos éticos, relacionados principalmente ao limite de atuação terapêutica e à ausência de autonomia por parte do paciente.

$\mathrm{Na}$ presente pesquisa foi possível constatar deficiências no conhecimento dos participantes acerca de cuidados paliativos e bioética, embora $80 \%$ da amostra tenha classificado a disciplina de bioética como extremamente relevante na graduação. A importância desse campo para a formação em fisioterapia é indiscutível, considerando que os conflitos éticos são inevitáveis, e é necessário que o profissional esteja preparado para lidar com eles $^{19-21}$. Entretanto, apesar dos avanços, a bioética ainda é embrionária nos cursos de fisioterapia ${ }^{24,25}$.

Quanto à discussão de conflitos éticos durante a graduação, $82 \%$ dos participantes consideraram essa prática extremamente relevante. Silva, Lima e Seidl ${ }^{19}$ confirmam essa importância, destacando, porém, a incipiência de tal debate na formação acadêmica do fisioterapeuta, principalmente no que diz respeito aos cuidados paliativos e à terminalidade da vida.

Ladeira, Silva Junior e Koifman ${ }^{26}$, em pesquisa com discentes de fisioterapia de uma universidade federal brasileira, apontam que para promover uma tomada de decisões mais direcionada e firme é essencial reconsiderar a formação acadêmica. No mesmo sentido, Santos ${ }^{27}$, em estudo com professores de instituições de ensino superior do estado do Rio de Janeiro, concluiu que a maioria dos docentes não chega a perceber problemas éticos relacionados com a confidencialidade ou privacidade dos indivíduos, observando apenas questões profissionais e deontológicas.

Com base nesses estudos, nota-se que o ensino superior em fisioterapia não contempla a formação ética dos discentes, permanecendo focado na aplicação de técnicas terapêuticas. No entanto, como aponta Bispo Júnior ${ }^{28}$, os modelos de formação profissional deveriam ser construídos a partir da relação entre questões sociais e conhecimentos fisioterapêuticos, e os cursos precisam contribuir para a transformação da sociedade.

Na presente pesquisa, quando perguntados se conseguiriam identificar e descrever algum conflito ético em sua prática, $72 \%$ dos participantes responderam sim, $24 \%$ que não, e $4 \%$ deixaram a questão sem resposta. Apenas $65 \%$ chegaram a de fato descrever um conflito ético, como solicitado. Seis (12\%) fisioterapeutas trouxeram o respeito à autonomia como conflito ético, e outros três (6\%) mencionaram os limites da atuação profissional, descrevendo situações de planos terapêuticos preestabelecidos por médicos. Dois (4\%) participantes descreveram ainda questões relacionadas à tomada de decisão, trazendo inquietudes quanto a deliberações não compartilhadas entre a equipe, além de pacientes e familiares. Outros dilemas mencionados foram a futilidade terapêutica relacionada a pacientes em cuidados paliativos, o problema de não falar a verdade para o doente e sua família (muitas vezes relacionado à dificuldade, ou até ao medo, de comunicar más notícias) e a falta de recursos financeiros do paciente. Duas falas demonstraram falta de compreensão acerca dos cuidados paliativos.

Santos e colaboradores ${ }^{29}$ analisaram o entendimento de enfermeiros, médicos, nutricionistas e fisioterapeutas sobre atendimento a pacientes em fim de vida em unidade de terapia intensiva oncológica. Os autores notaram fragilidades nessa compreensão, confirmando a percepção de que os profissionais sentem dificuldades para identificar dilemas éticos. A resultado semelhante chegaram Oliveira e Rosa ${ }^{30} \mathrm{em}$ estudo com enfermeiras de centro cirúrgico. Nesse caso, dilemas foram erroneamente 
entendidos como decisão (...) [em] situação vivenciada ${ }^{31}$, e conflitos como divergências de opiniões ${ }^{31}$.

Essa falta de compreensão sobre questões da bioética talvez tenha impactado o presente estudo - assim como em Renner, Goldim e Prati ${ }^{6}$ e Pereira ${ }^{8}-$, uma vez que a adesão à pesquisa foi pequena, e em muitos casos as respostas ao questionário se mostraram não pertinentes.

\section{Considerações finais}

Os fisioterapeutas que responderam aos questionários tinham, em média, cinco anos de formação, o que sugere maior interesse pela temática entre recém-formados. Das respostas obtidas, conclui-se que, para esses profissionais, a tomada de decisão e a discussão entre a equipe é muito importante na prática clínica. Percebem, no entanto, que não têm muita autonomia para decidir, visto que os pacientes já chegam com um plano decidido pelo médico.

Percebe-se a preocupação dos fisioterapeutas em respeitar a vontade do paciente e não adotar condutas que venham a fazer mal. Esses foram os principais critérios relatados para decidir a terapia do doente em processo de finitude. Nota-se, no entanto, certa dificuldade em descrever situações de conflitos éticos vivenciadas na prática. Observou-se, pelas respostas obtidas, que muitos dilemas estão ligados à falta de preparo técnico-científico dos profissionais, devida talvez a uma formação acadêmica deficiente. Os conflitos existem, permeiam a prática profissional, influenciam as condutas, mas são solucionados sem muita reflexão.

Os resultados reforçam a importância de formar profissionais capacitados para lidar com temas éticos. Essa importância é reconhecida pela maioria dos respondentes, que disseram considerar relevante que haja uma disciplina relacionada à bioética na graduação.

Os dados da pesquisa devem ser interpretados considerando suas limitações. Houve pouca adesão ao estudo por parte dos fisioterapeutas, seja em razão do tempo reduzido para responder o questionário (30 dias), seja porque os indivíduos ainda não estão acostumados a responder pesquisas por $e$-mail. Ademais, em nenhum momento foi descrito ou definido no questionário o que seria um conflito ou dilema ético. Se esse conceito tivesse sido explicado no instrumento, possivelmente o número de profissionais que conseguiriam descrever dilemas teria sido maior.

Os cuidados paliativos estão em ascensão no país, e a figura do fisioterapeuta tem sido destacada, reafirmando a importância da equipe multidisciplinar nesse tipo de assistência. Ainda assim, no entanto, há muito para evoluir nas discussões sobre cuidados paliativos e bioética, integrando esses conhecimentos desde o início da formação acadêmica.

Este artigo é resultado de dissertação de mestrado apresentada ao programa de Bioética, Ética Aplicada e Saúde Coletiva da Universidade Federal do Rio de Janeiro.

\section{Referências}

1. Gracia D. Pensar a bioética: metas e desafios. São Paulo: Loyola; 2010. p. 431.

2. O Conselho Regional de Fisioterapia e Terapia Ocupacional da $1^{\text {a }}$ Região. Crefito1 [Internet]. Quem somos; [s.d.] [acesso 14 fev 2020]. Disponível: https://bit.ly/3cEGGWO

3. Brasil. Lei $\mathrm{n}^{\circ}$ 6.316, de 17 de dezembro de 1975. Cria o Conselho Federal e os Conselhos Regionais de Fisioterapia e Terapia Ocupacional e dá outras providências. Diário Oficial da União [Internet]. Brasília, $18 \mathrm{dez} 1975$ [acesso 4 fev 2021]. Disponível: https://bit.ly/3ayWUNJ

4. Conselho Federal de Fisioterapia e Terapia Ocupacional. Resolução Coffito $n^{\circ} 424$, de 8 de julho de 2013. Estabelece o Código de Ética e Deontologia da Fisioterapia. Diário Oficial da União [Internet]. Brasília, n 147, $1^{\circ}$ ago 2013 [acesso 14 fev 2020]. Seção 1. Disponível: https://bit.ly/2L11YBB

5. Barnitt R. Ethical dilemmas in occupational therapy and physical therapy: a survey of practitioners in the UK National Health Service. J Med Ethics [Internet]. 1998 [acesso 14 fev 2020];24:193-9. Disponível: https://bit.ly/3mSLlj3 
6. Renner AF, Goldim J, Prati FM. Dilemas éticos presentes na prática do fisioterapeuta. Braz J Phys Ther. 2002;6(3):135-8.

7. Conselho Nacional de Saúde. Resolução CNS n 466, de 12 de dezembro de 2012. Aprova diretrizes e normas regulamentadoras de pesquisas envolvendo seres humanos. Diário Oficial da União [Internet]. Brasília, p. 59, 13 jun 2013 [acesso 14 fev 2020]. Seção 1. Disponível: https://bit.ly/38lbu4B

8. Pereira RAB. Dilemas éticos ligados à prática do fisioterapeuta no atendimento da criança com deficiência física ou mental [dissertação] [Internet]. São Paulo: Instituto Presbiteriano Mackenzie; 2006 [acesso 14 fev 2020]. Disponível: https://bit.ly/3b3G2RI

9. Instituto Nacional de Estudos e Pesquisas Educacionais Anísio Teixeira. Sinopse estatística da educação superior 2015 [Internet]. Brasília: Inep; 2016 [acesso 14 fev 2020]. Disponível: https://bit.ly/3dKM4VE

10. Guardia Mancilla P, Montoya-Juarez R, Marti-Garcia C, Herrero Hahn R, García Caro MP, Cruz Quintana F. Percepciones de los profesionales sobre la atención prestada, obstáculos y dilemas éticos relacionados con el final de la vida en hospitales, centros de atención primaria y residencias de ancianos. An Sist Sanit Navar [Internet]. 2018 [acesso 14 fev 2020];41(1):35-46. DOI: 10.23938/ASSN.0170

11. Instituto Brasileiro de Geografia e Estatística. Projeção da população do Brasil e das unidades da federação [Internet]. Rio de Janeiro: IBGE; 2018 [acesso 14 fev 2020]. Disponível: https://bit.ly/34SsZ10

12. Machado KDG, Pessini L, Hossne WS. A formação em cuidados paliativos da equipe que atua em unidade de terapia intensiva: um olhar da bioética. Bioethikos [Internet]. 2007 [acesso 14 fev 2020];1(1):34-42. Disponível: https://bit.ly/3rARidy

13. Rego S, Palácios M, Siqueira-Batista R. Bioética para profissionais de saúde. Rio de Janeiro: Editora Fiocruz; 2012.

14. Siqueira-Batista R, Gomes AP, Maia PM, Costa IT, Paiva AO, Cerqueira FR. Models of decision making in clinical bioethics: notes for a computational approach. Rev. bioét. (Impr.) [Internet]. 2014 [acesso 14 fev 2020];22(3):456-61. DOI: 10.1590/1983-80422014223028

15. Beauchamp T, Childress J. Princípios de ética biomédica. São Paulo: Loyola; 2002.

16. Beauchamp T, Childress J. Op. cit. p. 312.

17. Lorenzo CFG, Bueno GTA. A interface entre bioética e fisioterapia nos artigos brasileiros indexados. Fisioter Mov [Internet]. 2013 [acesso 17 mar 2021];26(4):763-75. DOI: 10.1590/S0103-51502013000400006

18. Pessini L, Bertachini L. Novas perspectivas em cuidados paliativos: ética, geriatria, gerontologia, comunicação e espiritualidade. Mundo Saúde [Internet]. 2005 [acesso 14 fev 2020];29(4):491-509. Disponível: https://bit.ly/2MlJoVl

19. Silva LFA, Lima MG, Seidl EMF. Conflitos bioéticos: atendimento fisioterapêutico domiciliar a pacientes em condição de terminalidade. Rev. bioét. (Impr.) [Internet]. 2017 [acesso 14 fev 2020];25(1):148-57. DOI: 10.1590/1983-80422017251176

20. Kovács MJ. Bioética nas questões da vida e da morte. Psicol USP [Internet]. 2003 [acesso 14 fev 2020];14(2):115-67. DOI: 10.1590/S0103-65642003000200008

21. Andrade BA, Sera CTN, Yasukawa SA. O papel do fisioterapeuta na equipe. In: Carvalho RT, Parsons HA. Manual de cuidados paliativos ANCP [Internet]. $2^{\text {a }}$ ed. São Paulo: Academia Nacional de Cuidados Paliativos; 2012 [acesso $1^{\circ}$ fev 2019]. p. 353-63. Disponível: https://bit.ly/2L4yFOx

22. Silva LFA, Lima MG, Seidl EMF. Op. cit. p. 149.

23. Oliveira T, Bombarda TB, Moriguchi CS. Physiotherapy palliative care in primary health care: theoretical essay. Cad Saúde Coletiva [Internet]. 2019 [acesso 14 fev 2020];27(4):427-31. DOI: 10.1590/ 1414-462x201900040166

24. Figueiredo AM. O ensino da bioética na pós-graduação stricto sensu, na área de ciências da saúde, no Brasil [tese] [Internet]. Brasília: Universidade de Brasília; 2009 [acesso 14 fev 2020]. DOI: 10.21713/ 2358-2332.2011.v8.213

25. Badaró AFV, Guilhem D. Bioética e pesquisa na fisioterapia: aproximação e vínculos. Fisioter Pesqui [Internet]. 2008 [acesso 14 fev 2020];15(4):402-7. DOI: 10.1590/S1809-29502008000400015 
26. Ladeira TL, Silva AG Jr, Koifman L. Fundamentos éticos na tomada de decisão de discentes de fisioterapia. Interface Comun Saúde Educ [Internet]. 2017 [acesso 14 fev 2020];21(62):675-85. DOI: 10.1590/ 1807-57622016.0273

27. Santos RNOL. O papel do docente na formação ética dos estudantes de fisioterapia: o olhar de quem ensina [dissertação] [Internet]. Rio de Janeiro: Fundação Oswaldo Cruz; 2016 [acesso 4 fev 2021]. Disponível: https://bit.ly/3oP2KzB

28. Bispo JP Jr. Formação em fisioterapia no Brasil: reflexões sobre a expansão do ensino e os modelos de formação. Hist Ciênc Saúde Manguinhos [Internet]. 2009 [acesso 14 fev 2020];16(3):655-68. DOI: 10.1590/ S0104-59702009000300005

29. Santos DCL, Silva MM, Moreira MC, Zepeda KGM, Gaspar RB. Planejamento da assistência ao paciente em cuidados paliativos na terapia intensiva oncológica. Acta Paul Enferm [Internet]. 2017 [acesso 14 fev 2020];30(3):295-300. DOI: 10.1590/1982-0194201700045

30. Oliveira MAN, Rosa DOS. Conflitos e dilemas éticos: vivências de enfermeiras no centro cirúrgico. Rev Baiana Enferm [Internet]. 2016 [acesso 14 fev 2020];30(1):344-55. DOI: 10.18471/rbe.v1i1.14237

31. Oliveira MAN, Rosa DOS. Op. cit. p. 344. 